\title{
Kidney cancer, ESMO 2016
}

\author{
Anil Kapoor, MD, FRCSC \\ Professor of Surgery (Urology), McMaster University, Hamilton, ON, Canada
}

Cite as: Can Urol Assoc J 2016;10(11-12Suppl6):S227-30.

http://dx.doi.org/10.5489/cuaj.4282

\section{Sunitinib as adjuvant treatment}

The S-TRAC study was designed to determine whether adjuvant sunitinib could improve outcomes among patients postresection of clear-cell, loco-regional renal cell carcinoma (RCC) at high risk of disease recurrence $(\geq \mathrm{T} 3$ and/or $\mathrm{N}+$, with any Fuhrman's grade and any Eastern Cooperative Oncology Group [ECOG] performance status [PS]). ${ }^{1}$ The results were simultaneously published in the New England Journal of Medicine. ${ }^{2}$

The study included 615 patients with no history of prior systemic therapy. Patients were randomized to receive either oral sunitinib $50 \mathrm{mg} /$ day (on a four-weeks-on/two-weeks-off schedule) or placebo for one year, or until recurrence, second cancer, significant toxicity, or withdrawal of consent. The primary efficacy outcome was disease-free survival (DFS) by independent central review. Secondary analyses included overall survival (OS) and subgroup analysis of the primary outcome in higher-risk populations.

As shown in Fig. 1, adjuvant sunitinib was associated with improvements in median DFS vs. placebo (6.8 years vs. 5.6 years, hazard ratio $[\mathrm{HR}] 0.761 ; \mathrm{p}=0.03)$, as well as three-year DFS (64.9\% vs. $59.5 \%)$ and five-year DFS (59.3\% vs. $51.3 \%)$.

Among patients considered to have higher-risk disease (1: T3, N0 or Nx, M0, Fuhrman's grade $\geq 2$, ECOG PS $\geq 1$; 2: T4, N0 or Nx, M0, any Fuhrman's grade and any ECOG PS; or 3: any T, N1-2, M0, any Fuhrman's grade and any ECOG PS), the improvement in median DFS was similar to the overall population: 6.2 years for adjuvant sunitinib and 4.0 years for placebo (HR 0.737; $p=0.044)$. OS data were not yet mature at the time of data cutoff.

Grade 3 treatment-emergent adverse events (AEs) were observed among $48.4 \%$ of sunitinib-treated patients and $15.8 \%$ of placebo-treated patients. Grade $4 \mathrm{AE}$ rates were $12.1 \%$ and $3.6 \%$ for sunitinib and placebo, respectively. The proportions of patients who discontinued due to AEs were $28.1 \%$ for sunitinib and $5.9 \%$ for placebo.

This new data gives new options for those patients postresection at high risk for recurrent kidney cancer. The standard of care has been close surveillance of those patients at high risk for development of metastatic disease (roughly one-third of our patient population of resected kidney cancer), with initiation of tyrosine kinase inhibitor or clinical trial once metastatic disease has developed. Now, patients with high-risk resected disease can consider the option of adjuvant sunitinib, with DFS advantage. We look forward to OS results in due course.

\section{New research with cabozantinib}

The CABOSUN study compared oral cabozantinib $60 \mathrm{mg}$ daily for six-week cycles to oral sunitinib $50 \mathrm{mg}$ daily in a four-week-on/two-week-off schedule among 157 patients with advanced RCC with a clear-cell component, measurable disease, and no prior experience with systemic therapy. ${ }^{3}$ The primary efficacy endpoint was progression-free survival (PFS); secondary endpoints included OS, objective response rate (ORR), and safety in this first-line trial.

As shown in Fig. 2, cabozantinib was associated with a significant improvement in median PFS compared to sunitinib (8.2 months vs. 5.6 months; HR 0.69; $p=0.012$ ). The PFS analysis also favoured cabozantinib in pre-specified subgroups (IMDC intermediate- or poor-risk groups with or without bone metastases).

The ORR was $45.6 \%$ for cabozantinib (one complete response) and $17.9 \%$ for sunitinib (one complete response). Sixty-nine of the $79(87.3 \%)$ cabozantinib-treated patients experienced tumour reduction, compared to 34 of the 78 sunitinib-treated patients $(43.6 \%)$.

There was no significant difference between groups in OS (median 30.3 months for cabozantinib and 21.8 months for sunitinib; HR 0.80; 95\% confidence interval [Cl] 0.50-1.26).

The proportions of patients experiencing a Grade 3 or 4 AE were similar in each group: $65 \%$ for cabozantinib and $68 \%$ for sunitinib.

This phase 2 trial suggests that cabozantinib may have benefit in the first-line treatment of metastatic RCC as an 


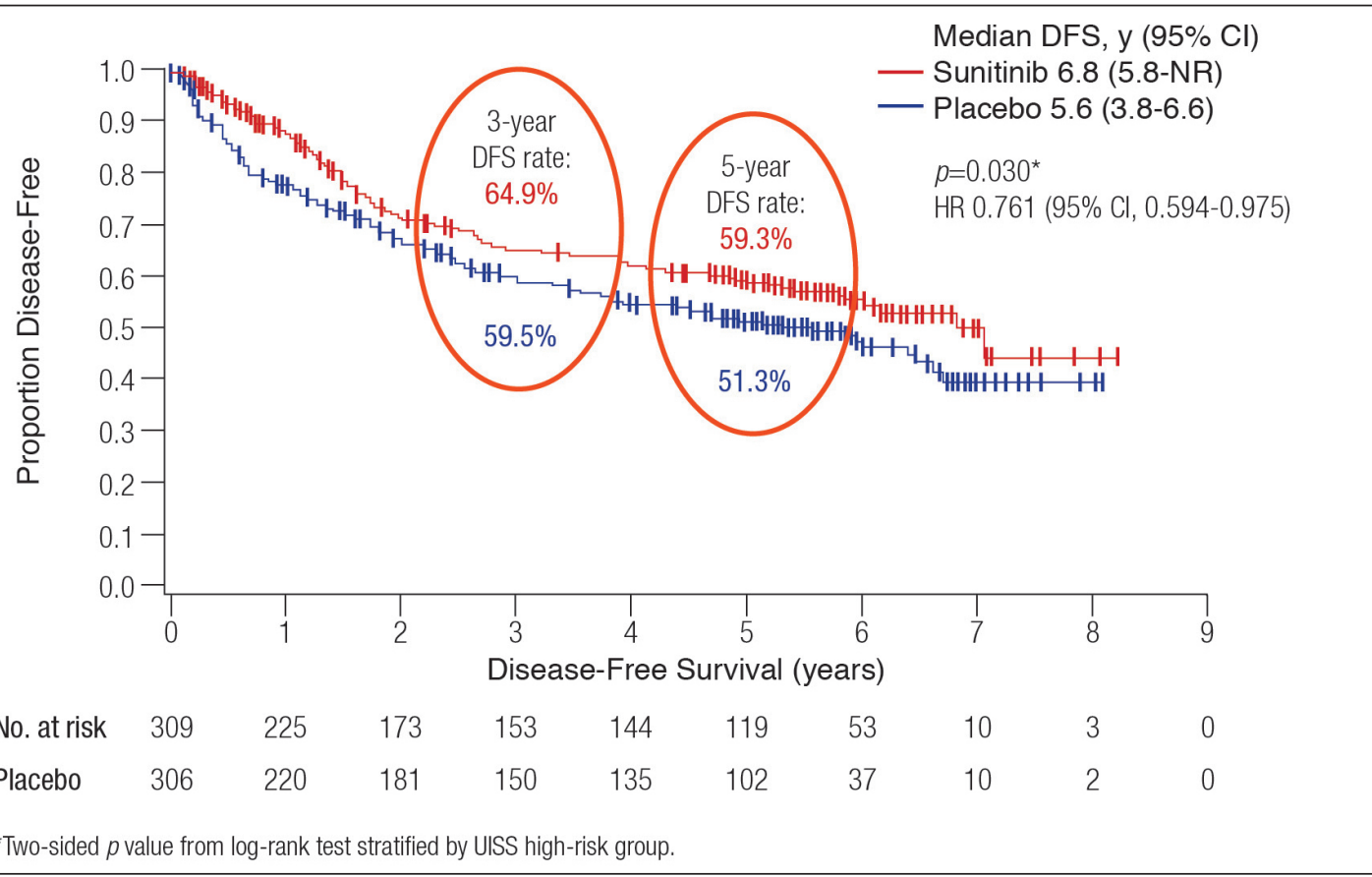

Fig. 1. Disease-free survival: Adjuvant sunitinib vs. placebo in high-risk renal cell carcinoma (S-TRAC study). CI: confidence interval; DFS: disease-free survival; HR: hazard ratio.

alternative to current first-line options of sunitinib, pazopanib, and bevizumab/interferon.

Additional information on cabozantinib from the phase 3 METEOR study in advanced RCC were also presented during ESMO 2016.4,5 The primary results of METEOR demonstrating superiority of cabozantinib vs. everolimus for PFS and OS were previously published. ${ }^{6,7}$ In new analyses presented at ESMO 2016, researchers showed that these advantages were observed irrespective of tumour burden or metastatic sites, and that the safety of cabozantinib was similar for those with high or low tumour burden. ${ }^{4}$

Additionally, quality of life analyses using the FACT Kidney Symptom Index (FKSI-19) and EuroQol (EQ-5D-5L) showed that maintenance of quality of life over the METEOR

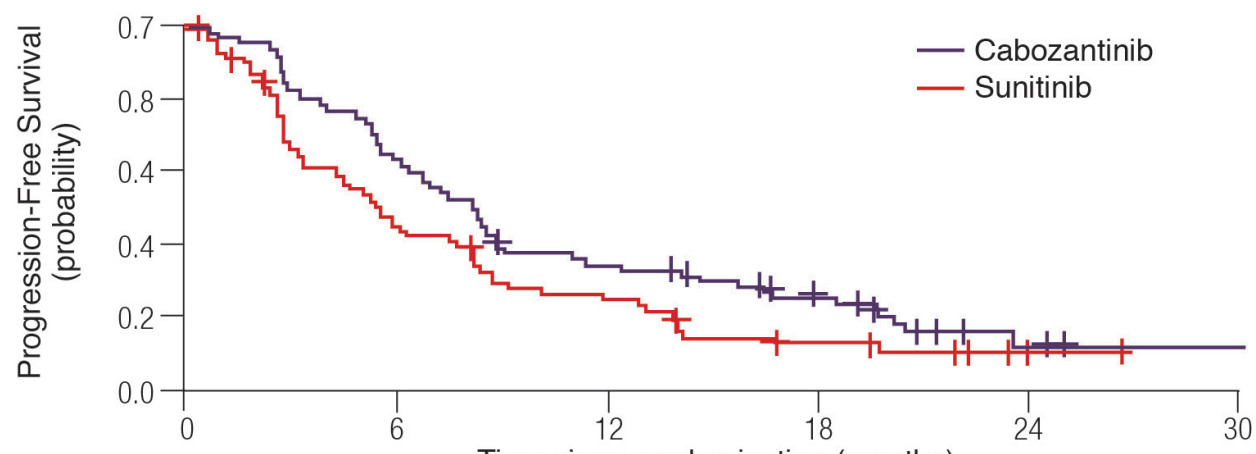

${ }^{*}$ Adjusted for bone metastases and IMDC risk group.

Fig. 2. Progression-free survival for cabozantinib vs. sunitinib in advanced renal cell carcinoma (CABOSUN study). Cl: confidence interval; HR: hazard ratio; PFS: progression-free survival. 


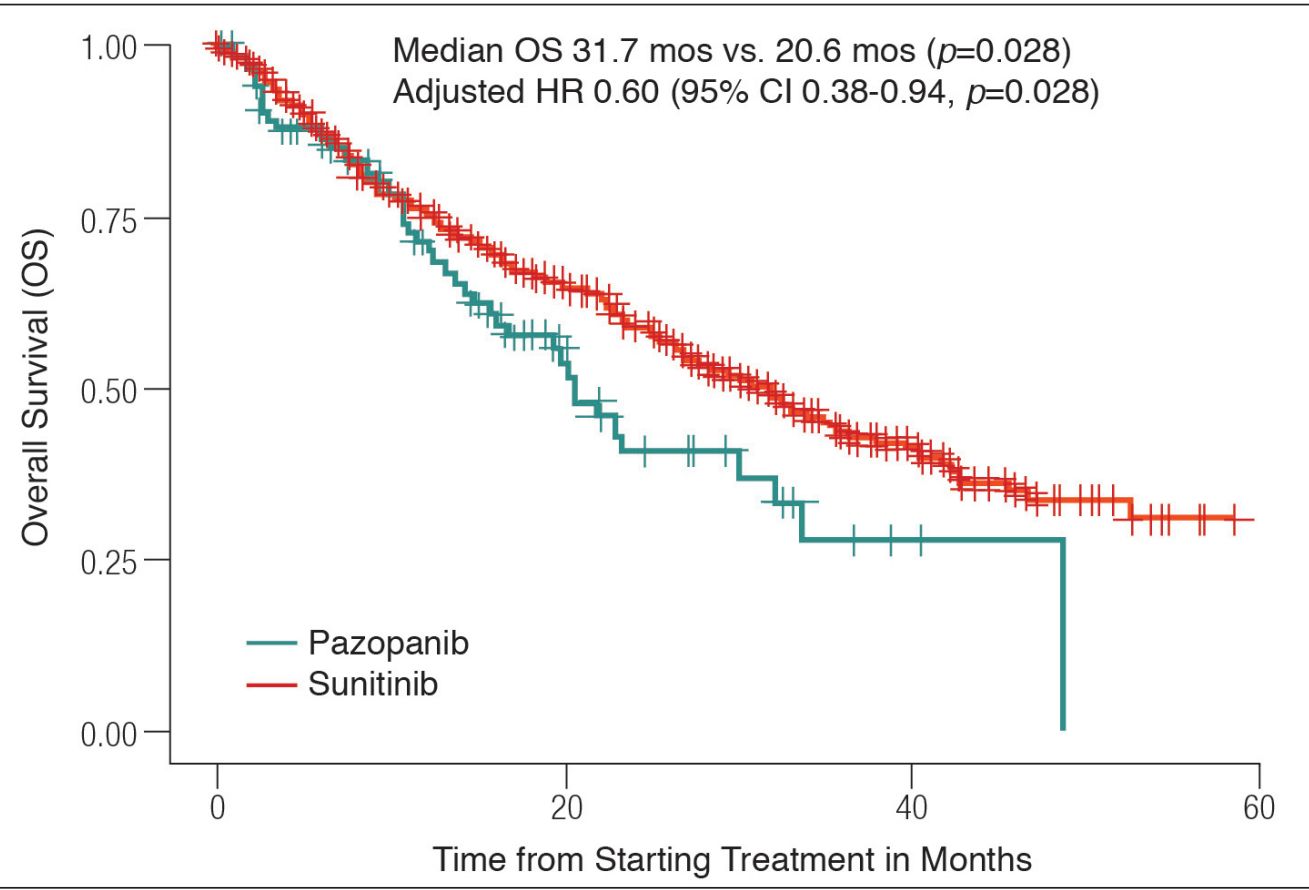

Fig. 3. Overall survival with sunitinib or pazopanib for first-line treatment of metastatic renal cell carcinoma: Real-world experience in Canada. Cl: confidence interval; HR: hazard ratio; OS: overall survival.

study period was similar in the cabozantinib and everolimus groups. ${ }^{5}$ However, time to deterioration - defined as death, progressive disease or $\mathrm{a} \geq 4$-point deterioration in the FKSI-DRS (nine-item) — was significantly longer with cabozantinib than with everolimus (median 5.5 months vs. 3.7 months; $p<0.0001){ }^{5}$

\section{Real-world experience with sunitinib and pazopanib in Canada}

Canadian researchers presented an analysis of data from the Canadian Kidney Cancer Information System (CKCis) database evaluating outcomes and comparing dose-modifying toxicities with first-line sunitinib $(n=577)$ or pazopanib $(n=93)$ in patients with clear-cell metastatic RCC. ${ }^{8}$ The key analyses were OS, time to treatment failure (TTF), and dosemodifying toxicities.

As shown in Fig. 3, the median OS was significantly longer for sunitinib compared to pazopanib (31.7 months vs. 20.6 months; adjusted HR 0.60; $p=0.028)$. There was no significant difference in TTF (11.0 months for sunitinib vs. 8.4 months for pazopanib; $\mathrm{p}=0.130$; adjusted HR 0.87 [95\% Cl 0.59-1.28). A limitation of this study includes the small numbers in the pazopanib arm.

There were significant differences in the incidence of some dose-modifying toxicities. Those that were significantly more common with sunitinib than pazopanib were mucositis $(16.3 \%$ vs. $7.5 \%$; $p=0.028)$, hand-foot syndrome $(12.0 \%$ vs. $3.2 \% ; p=0.01)$, and gastroesophageal reflux disease $(6.6 \%$ vs. $1.1 \%$; $p=0.031$ ). Liver toxicity/altered liver enzymes was significantly more common with pazopanib than sunitinib $(14.0 \%$ vs. $2.6 \% ; p=0.001)$.

\section{Impact of modification of the sunitinib dosing schedule}

At ESMO 2016, researchers presented data evaluating the impact of changing the sunitinib dosing schedule from four weeks on, two weeks off to two weeks on, one week off among 130 patients who experienced treatment-emergent AEs on the initial therapy. ${ }^{9}$ The subjects had a median of two cycles (range 1-56) for the initial four/two-week schedule (344 total cycles) and a median of six cycles (range 1-18) with the two/one-week schedule (1273 total cycles).

The median PFS for this cohort was 13.5 months $(95 \% \mathrm{Cl}$ 12-17) and median OS was 30.0 months (95\% Cl 24-36). The investigators noted that this compared favourably to the median durations previously reported in sunitinib trials (PFS 11.0 months and OS 26.4 months), where the usual practice was to reduce the sunitinib dose and maintain the four/two-week schedule.

\section{Immuno-oncology combined with axitinib: Preliminary data}

Two phase $1 \mathrm{~b}$ studies evaluating the safety of combining an immuno-oncology agent with targeted therapy for the firstline treatment of mRCC were presented at ESMO 2016.10,11 The immuno-oncologic agents were the PD-1 inhibitor pem- 
brolizumab ${ }^{10}$ and the PD-L1 inhibitor avelumab. ${ }^{11}$ Both studies included oral axitinib $5 \mathrm{mg}$ twice daily.

The pembrolizumab study included 52 patients with confirmed clear-cell, advanced RCC. The dose-finding phase of the study identified pembrolizumab $2 \mathrm{mg} / \mathrm{kg}$ IV every three weeks as the optimal dose for the dose-expansion phase of the study.

Thirty-four patients (65.4\%) experienced a Grade 3 or higher treatment-emergent AE. Nine patients (17.3\%) discontinued due to AEs. There were no new or unusual toxicities reported during followup. Among the 52 patients treated with axitinib + pembrolizumab, 49 (94.2\%) experienced tumour shrinkage and 37 (71.2\%) achieved objective response (three complete responses and 34 partial responses). PFS, OS, and response duration data were not yet mature. Using the latest available data, however, median PFS was 15.1 months.

The avelumab study included only six patients at the time of data collection. ${ }^{11}$ The dose-finding segment of the study identified avelumab $10 \mathrm{mg} / \mathrm{kg}$ IV every two weeks as the dose to be used moving forward. Among the six patients studied to date, four developed a Grade 3-4 treatmentemergent $\mathrm{AE}$ and one patient discontinued due to AEs. All six patients had a partial response to the avelumab-axitinib combination. This study is ongoing and had accrued 54 patients as of September 13, 2016.

\section{References}

1. Ravaud A, Motzer RJ, Pandha HS, et al. Phase 3 trial of sunitinib vs. placebo as adjuvant treatment for high-risk renal cell carcinoma after nephrectomy. Presented at ESMO 2016; Presentation \#LBA11_PR.

2. Ravaud A, Motzer RJ, Pandha HS, et al. Adjuvant sunitinib in high-risk renal cell carcinoma after nephrectomy. N Engl J Med 2016; Oct 9 [epub ahead of print]. https:/doi.org/10.1056/NEJMoal611406

3. Choueiri TK, Halabi S, Sanford B, et al. CABOzantinib vs. SUNitinib (CABOSUN) as initial targeted therapy for patients with metastatic renal cell carcinoma (mRCC) of poor and intermediate risk groups: Results from ALLIANCE A031203 trial. Presented at ESMO 2016; Presentation \#LBA30.

4. Powles T, Escudier B, de Souza P, et al. Efficacy of cabozantinib vs. everolimus by metastatic site and tumour burden in patients with advanced renal cell carcinoma in the phase 3 METEOR trial. Presented at ESMO 2016; Poster \#814P.

5. Cella D, Escudier B, Tannir NM, et al. Quality of life in the phase 3 METEOR trial of cabozantinib vs. everolimus for advanced renal cell carcinoma. Presented at ESMO 2016; Poster \#816P.

6. Choueiri TK, Escudier B, Powles T, et al. Cabozantinib vs. everolimus in advanced renal cell carcinoma. N Engl J Med 2015;373:1814-23. https:/doi.org/10.1056/NEJMoa1510016

7. Choueiri TK, Escudier B, Powles T, et al. Cabozantinib vs. everolimus in advanced renal cell carcinoma (METEOR): Final results from a randomized, open-label, phase 3 trial. Lancet Oncol 2016;17:917-27. https:/doi.org/10.1016/S1470-2045(16)30107-3

8. Lalani A, Li H, Heng D, et al. Real-world outcomes of patients with metastatic renal cell carcinoma using first-line sunitinib or pazopanib: The Canadian experience. Presented at ESMO 2016; Poster \#819P.

9. Gyergyay F, Budai B, Nagyivanyi K, et al. Sunitinib (two weeks on/one week off schedule) in metastatic renal cell cancer patients. Progression-free and overall survival. Presented at ESMO 2016; Poster \#820P.

10. Atkins MB, Plimack ER, Puzanov I, et al. Axitinib in combination with pembrolizumab in patients with advanced renal cell carcinoma: Preliminary safety and efficacy results. Presented at ESMO 2016; Poster \#773PD.

11. Larkin J, Rini BI, Nathan P, et al. Phase Ib dose-finding study of avelumab (anti-PD-LI) + axitinib in treatment-naive patients with advanced renal cell carcinoma. Presented at ESMO 2016; Poster \#775PD. 